\title{
THE TREATMENT OF BURNS BY THE CLOSED-PLASTER METHOD, WITH CERTAIN PHYSIOLOGICAL CONSIDERATIONS IMPLICIT IN THE SUCCESS OF THIS TECHNIQUE
}

\author{
By WILLIAM W. L. GLENN, ${ }^{1}$ HELEN H. GILBERT, aNd CECIL K. DRINKER \\ (From the Department of Physiology, Harvard School of Public Hcalth, Boston)
}

(Received for publication May 11, 1943)

\section{THE PROBLEM}

The prime difficulty encountered in the treatment of severe burns, and the greatest factor involved in causing later obstacles to successful healing, is the early and frequently persistent increase in the permeability of the blood capillaries. The reasons which cause us to make so categorical a statement are as follows:

The loss of plasma from dilated and injured capillaries may be disastrous in several ways. One of these, the systemic effect, is so widely appreciated as to have dominated physiological thinking in the treatment of burns almost to the exclusion of other principles very vital to efficient

I therapy. There can be no doubt that reduction in plasma volume by leakage from abnormal capillaries in burned regions and possibly a slower but obstinately insidious leakage from capillaries all over the body may and often does cause surgical shock and death. If, as in our experiments, the feet of an anesthetized dog are burned quantitatively by immersion in very hot water for precise periods of time (Glenn, Peterson, and Drinker (1)), the amount of plasma that may be lost into the subcutaneous tissue without leakage from the surface of the skin has not been sufficiently appreciated. Underhill et al. (2) and Harkins (3) called attention to how great this loss may be. Following an experimental burn such as we have utilized, edematous swelling may spread though the subcutaneous tissue for a considerable distance-especially so after the lymphatics draining this area become blocked. Indeed, in one animal with the 2 front feet severely burned and with the exudate spreading beyond the burned area, there was a loss of $1 / 3$ the total blood volume into the subcutaneous tissue from the toenails to the humero-radio-ulnar joints. To combat this loss

1 First Lieutenant, Medical Corps, Army of the United States. of fluid from the blood into the tissues, and in man the difficulty is usually greatly increased by free weeping of fluid from denuded surfaces, blood plasma is given intravenously and often in very large amounts. This is physiological therapy and, as such, its use should be accompanied by physiological thinking. The object in view is the maintenance of blood volume at a level which will assure reasonably normal distribution of the circulating blood. The vaso-constriction which accompanies loss of blood represents an attempt to hold blood pressure at a level providing adequate supply to vital parts of the body. The vascular bed undergoes reduction in size and relatively nonessential areas, such as the skin, subcutaneous tissue, and muscles, may even be subjected to anoxia through the struggle of the organism to sustain some sort of blood supply to regions where deprivation of blood spells death. It should be realized that the local vaso-dilator reaction in and around a burn causes the region to become an open shunt with capacity to contain practically a maximal amount of intravascular blood under local conditions of pressure and flow, comparable to those normal for the tissues involved when they are operating at highest functional capacity. A large burn is thus a suddenly opened reservoir into which the patient loses blood intravascularly. But, in addition, the walls of the reservoir are leaky, so that the amount of fluid entering the affected region is more than that returning from it to re-enter the general circulation. Intravenous infusion of plasma is the essential resource in such a state of affairs. At the same time, it is clear that no good can be done if the plasma is given so rapidy that for a time the pressure is raised above a reasonable normal level for a prone, quiescent patient. Invariably, the injured and inflamed area will receive an undue share of the fluid which is given. Furthermore, leakage of plasma through the damaged capillary walls will be directly pro- 
portional to the flow and pressure of the blood, and consequently, one can count upon losing plasma rapidly if the infusion is intemperately hasty or unduly large in quantity. From the physiological point of view, plasma replacement would be best fitted to the needs of the situation if supplied through occasional small injections, or as an intravenous drip. Obviously, amongst the aims of local therapy are restraint of vascular dilatation in the part and restraint of leakage from the injured capillaries, leakage which will be abnormally great with the vessels at ordinary diameter and increased surprisingly if they are dilated. Given time, many of the capillaries involved in a burn recover normal permeability and diameter and it should be within one's efforts in treating burns to hasten the restoration of normal capillary function as rapidly as possible. We do not know just where fluid escapes from capillaries, whether through the endothelial cells, between them, or universally. Nor do we know that continued abnormal leakage from capillaries of itself tends to sustain leakiness. But anyone with experience in one of the most exacting techniques used in physiological experiment, the perfusion of isolated mammalian tissues, knows that once edema begins to appear it is extraordinarily difficult or impossible to stop it. It is thus quite reasonable to suggest that the more we impose or permit conditions which enhance leakage from damaged capillaries, the more we may promote persistence in leakage and postpone recovery of the great desideratum, a vascular bed of normal capillaries.

These remarks upon the intelligent use of intravenous plasma apply even more forcibly to the employment of simple solutions, saline or glucose. Not only will such intravenous injections, by dilution of plasma proteins, enhance leakage from the vessels in the burn, but Blalock et al. (4) have shown that they actually wash proteins out of the blood. Water by mouth and salt solution subcutaneously, or even intraperitoneally, may be resorted to when plasma is not available, or with plasma, since in such cases fluid will be taken up by the blood stream as needed and not in excess, but nothing could be better designed to promote the excessive permeability of capillaries injured by heat than large intravenous injections of simple solutions.

In addition to the loss of plasma from the capillaries in burns and the systemic effect resulting from lowered blood volume, there is a second circumstance localized in the burned region attending this loss which has not received enough attention. It is the fact that when plasma leaves capillaries and is distributed through injured tissues, coagulation sooner or later occurs. The necessity for clotting as an early event in the healing of wounds is so great that we forget excess coagulated plasma in the tissues is in reality a foreign body which must be removed and the amount of coagulated exudate in and around burned tissue may be very great indeed. Physicians and surgeons are prone to look upon swelling as a symptom to be disposed of by attacking its cause or the general condition of which it is apparently an inevitable part. But swelling means abnormal tissue environment. In burns, excessive permeability of the capillaries causes outpouring of plasma into tissues which at the time are in various stages of heat coagulation or injury. Irrespective of the severity of the burn, there is a transition from dead coagulated tissue protein at the surface of heat contact inward through cells less and less affected until uninjured tissue is reached. Under normal circumstances, blood flowing through the capillaries maintains the even temperature of the tissues and during the application of heat, the stream of capillary blood is an important means of resisting the rise in temperature and also the blockage of vessels with plasma and cells coagulated by heat. Even in tissue killed by a burn, capillaries here and there still conduct blood, and through the injured walls of these vessels, plasma leaks freely out amongst tissue cells which are not mutilated but are dead and must be removed during the healing process. All over the body and notably in the skin, which is the part most often burnt, the capillaries exist as a complicated net into which blood is poured from the arterioles. The first effect of heat, sufficiently great to cause injury, is apparently direct stimulation of arterioles and capillaries which contract, so that for a short time the burned region may become pale. But very promptly, and brought about through axone reflexes confined to the burned and immediately adjacent areas, capillaries, arterioles, and arterio-venous anastomoses dilate widely. This increase in local blood supply may encounter many capillaries which contain 
injured or even broken-down red cells, together with blood proteins coagulated by heat. Swelling opens lymphatics and promotes lymph drainage. As the exudate accumulates, the tissues are spread apart and the inflammatory edema extends beyond the burned region, the excess tissue fluid, which is under very considerable pressure, acting as a sort of wedge which opens more and more space to be filled by fresh fluid leaking rapidly through the injured walls of the capillaries. The extension of swelling is very evident in Figure 1. After some hours, lymph flow begins to subside due, apparently, to the fact that fibrin formation becomes well established in the tissue fluid and in the lymph within the lymphatics. Menkin (5) summarized his ideas upon the relation of tissue destruction to the spread of inflammation, attributing the check in spread to the effectiveness of fibrin formation, which, in turn, was dependent on the release of thromboplastic material from damaged tissue cells to promote the formation of thrombin in the inflammatory exudate and the reaction with fibrinogen to form fibrin. Evidently, heat coagulation of tissues does not result in cell changes

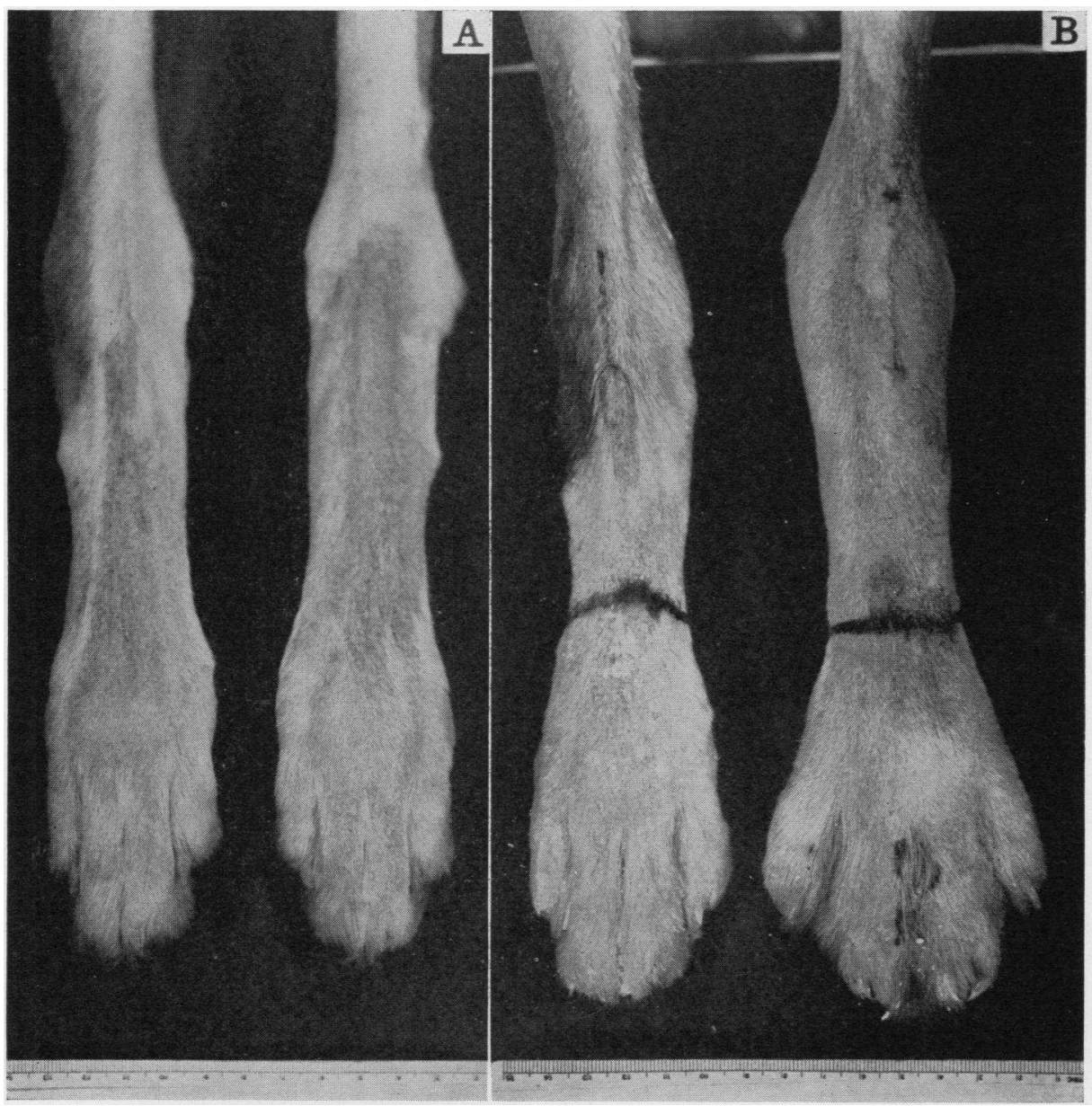

Fig. 1. Swelling After an Experimental Burn

A. Feet of anesthetized dog just before burning. B. Twenty-four hours after the burn. The black lines indicate the depth to which the feet were immersed for 30 seconds in water at $100^{\circ}$ C. A plaster cast was applied to the right paw, the upper level being about $3 \mathrm{~cm}$. above the black line.

No treatment of any sort was used upon the left. Note the spreading of the toes in this paw and the extension of edematous swelling to the ankle joint. As often happens, blisters formed between the toes and produced weeping points through which fluid was steadily lost. 
which at once liberate thromboplastin, as is the case in mutilating injuries, and fibrin formation is slow.

In a burn, uncomplicated by other trauma, conditions are thus particularly favorable for the eventual accumulation of a large amount of coagulated plasma in the tissues. For some time after the burn, fluid pours out of the capillaries and, though it is contained in dead tissue, there is something about the effect of heat which does not result in prompt formation of fibrin. It is as if one had to wait for lytic processes to break down dead cells before fibrin formation becomes noteworthy. Thromboplastin seems to arise slowly, so that the burned part which is saturated with exudate gradually is filled not with fluid but with a fibrinous gel almost as stiff as may be obtained from whole blood, since the protein content of the tissue fluid from burned areas may reach a concentration of 5.5 per cent-almost that of blood. Furthermore, since the presence of an extravascular gel does not restrain capillary leakage until the lymphatics and tissues are filled to capacity with it, fibrin formation is a progressive and persistent phenomenon, leaving the victim with a gratuitous mass of interstitial foreign material which, with the heat-killed tissue, must be removed before healing is complete. In our opinion, extensive fibrin formation in and about burns is a decided obstacle to recovery. It provides a fixed and abundant culture medium for bacterial growth and this promotes the destruction of tissue which accompanies infection. Even if uninfected, it induces intensive fibrous tissue formation as healing progresses. Obviously, excessive permeability of injured capillaries is responsible for the situation.

From our point of view, the problem in treating burns, which dominates all else during the first week following the accident, consists in finding some way of controlling abnormal loss of plasma from the capillaries. There are other factors to be considered, such as the formation of a toxin or toxins in the burned tissue and their distribution through the body, but even in relation to this still unsettled possibility in burns, the free accumulation of a large amount of exulate may readily promote absorption. In the face of a severe burn, if one could check capillary leakage and at the same time keep the circulation in the part so normal as to provide the best chance for recovery, one would possess the best means of dealing with the injury. To us, it seems that the closed-plaster treatment, as used in the experimental burns we shall describe, is illustrative of a method which operates so as to restrain the unfortunate occurrences we have outlined and, at the same time, retains the part in good physiological condition. We have been concerned with the exposition of principles. How to make use of the closed-plaster technique or some variant of it in the different types and sites of human burns are problems for gradual exploration in the clinic, since they can be little more than matters for conjecture in the laboratory.

\section{EXPERIMENTAL METHODS}

Glenn, Peterson, and Drinker (1) called attention to the necessity for a standard procedure in the production of experimental burns and their simple method of burning was used in all of these experiments. The animals were anesthetized with nembutal and a paw was immersed to the desired depth, for a definite length of time, in water heated to a definite temperature. Lymph, when desired, was collected by cannulation of a lymphatic just above the burn, as described in the first communication.

Two groups of experiments upon the use of the closedplaster treatment were carried out.

The first group was designed to test the patency of the circulation beneath the plaster cast. In this group, dental plaster, with a hardening time of 5 to 7 minutes when mixed with $60^{\circ} \mathrm{F}$. water, was poured into a container and the burned paw was inserted into the liquid plaster immediately after the burn, care being taken to bring the plaster a distance of 2 to $3 \mathrm{~cm}$. above the upper margin of the burn. Studies were then carried out as desired. Figure 2 (B, C, and D) shows a dog's paw encased in dental plaster after burning. Casting in this manner results in perfect encasement of the part without exertion of pressure.

In the second group, the experiments were designed to test the efficacy of this method in the treatment of burns. Two methods of application of plaster were employed: (1) The skin-tight dental plaster applied as described above. As soon as the plaster hardened, the solid-walled container was slipped off, the plaster dried thoroughly under an electric fan, and a perforated-walled metal container slipped on over the dried plaster. This second metal cover was necessary in order to prevent cracking and loss of the plaster as the dog moved about. It was held in place on the cast by a few dabs of water-proof glue. Upon awakening from the anesthetic, the dog would start to walk at once upon the metal container without any apparent discomfort. The skin of the leg at the upper part of the cast was protected from the sharp edge of the plaster by a small collar of gauze incorporated in 


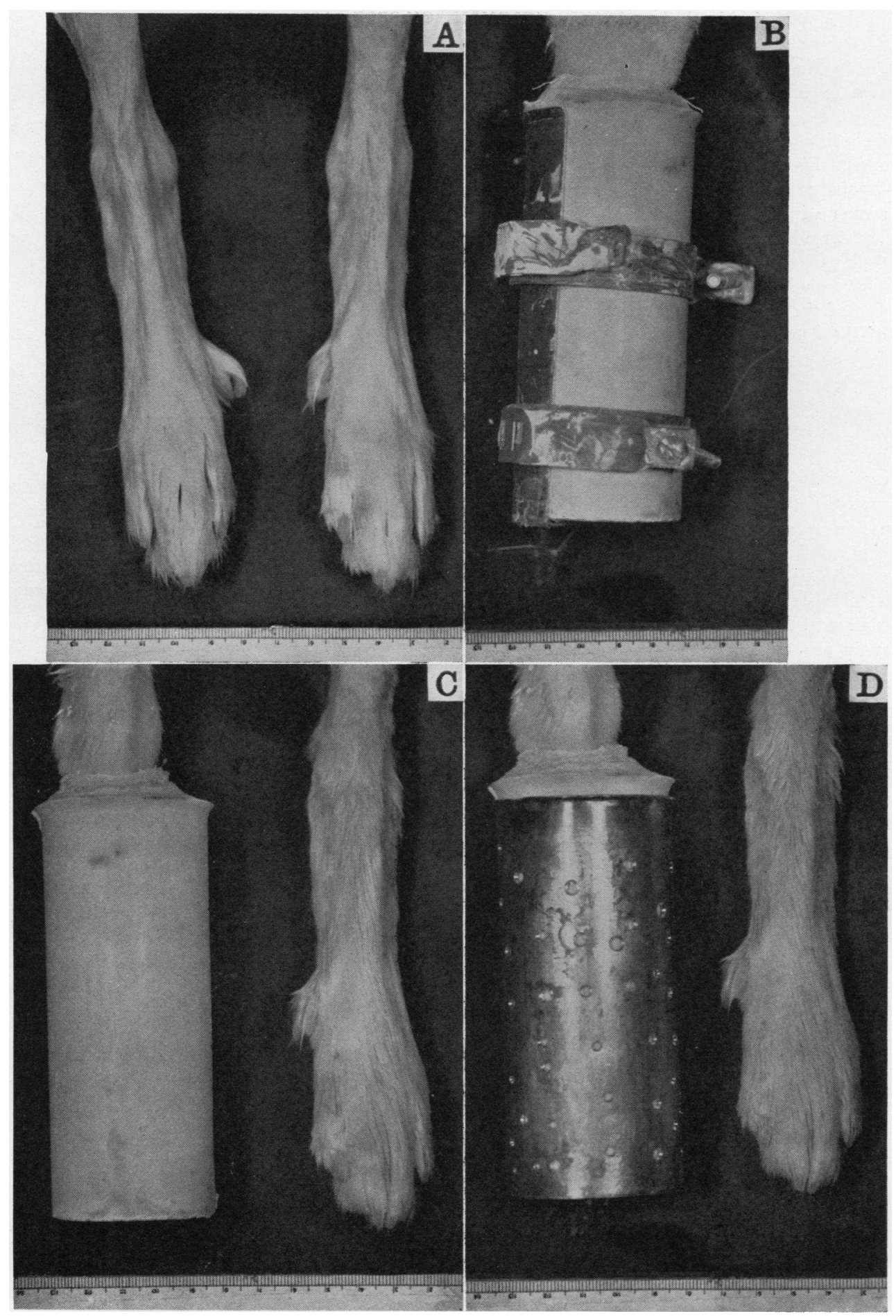

Fig. 2. Application of a Plaster Cast to a Burned Paw

A. Hind feet of an anesthetized dog, prior to burning. B. The right foot has been burned by immersion in hot water and immediately after removal, was thrust into a brass can containing liquid dental plaster. This can was in 2 halves, held tightly together by hose clamps. In the illustration, the plaster has hardened and $1 / 2$ of the can has been removed. C. The casted foot after removal of the can and ready for thorough drying by means of an electric fan. D. A perforated can to permit further evaporation from the plaster has been cemented in place in order to protect the cast as the animal moves about, following recovery from anesthesia. Note the gauze collar at the top of the plaster to prevent chafing. 
the plaster. (2) Another group of dogs was treated by the application of a roller-plaster bandage over 2 or 3 layers of very thin, dry, sterile gauze. The application of the gauze prior to the roller plaster was done for several reasons. First, with the relatively thin shell of plaster as compared to the skin-tight dental plaster, it offered a means of absorbing and localizing the small amount of leakage that one sees even after the application of a closed-plaster dressing. Second, it provided sufficient padding to allow for minor differences of fit in the application of the roller bandage, a factor not present in the use of the dental-plaster cast. It is to be stressed that absolutely no pressure was used in either the gauze or plaster bandage application. The closed-plaster technique is not a compression or pressure treatment. It is simply a means of holding the part exactly to the volume present when the plaster is applied. This aim is best attained by casting the part in liquid plaster, but the unwieldy character of the cast and the difficulty of removal made the use of plaster bandage imperative and if care is used during application, there is no need to build up pressure as successive turns are put in place. Following application, the roller-plaster bandage is thoroughly dried and the dog allowed to recover from the anesthesia.

The casts or plaster bandages were left in place for varying periods. In some cases, they were removed and replaced at intervals of 24 to 48 hours to watch the progress of the wound. In most instances, however, they were left undisturbed for 10 to 14 days.

\section{RESULTS}

\section{Experiments in Group 1}

Those designed to display the condition of the circulation in the burned and casted foot.

A. Drainage of lymph. As has been previously pointed out, following a burn the capillaries are widely dilated and plasma is lost through them into the subcutaneous tissue. The lymph flow is immediately augmented, reaching a peak within a few hours, then falling off as blockage of the extravascular tissue spaces and lymphatics is accomplished by clotting of the exudate. Figure 3 shows the lymph drainage from casted and noncasted front feet, burned for 30 seconds by immersion in water at $100^{\circ} \mathrm{C}$. Curves 1,1 , represent the protein concentration and lymph flow from the right front paw. No cast was applied. Curves 2, 2, are the same for the left front paw, burned identically but put into dental plaster immediately after the burn. The first arrow indicates the time of burning and at arrow prime, the cast was removed from the left front paw. These curves indicate that although lymph drainage was less in the casted foot, the shape of the curve of

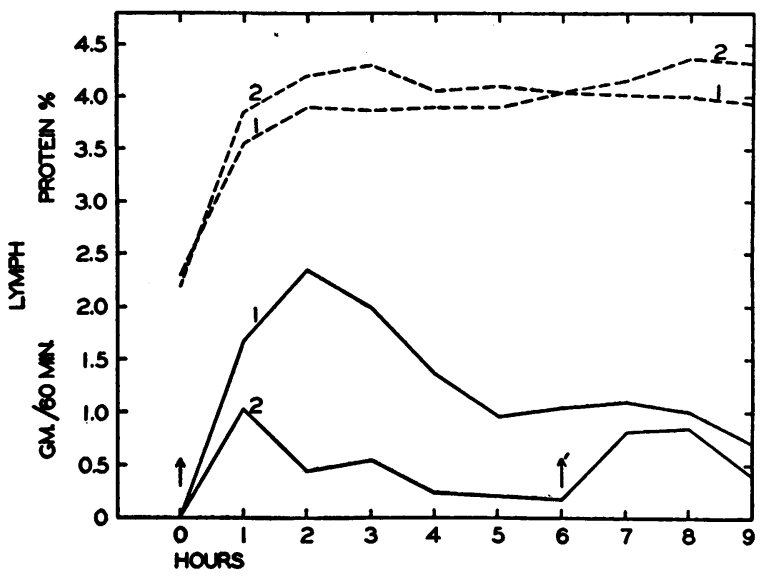

Fig. 3. Lymph Flow and Lymph Protein Content from Casted and Uncasted Burned Front Feet in an Anesthetized Dog

The upper pair of broken lines show the protein concentration in per cent from the 2 feet and the lower solid lines the lymph flow in grams per 60 minutes.

Curves 1 and 1 are from the right front paw where no cast was applied and curves 2 and 2 from the casted left foot.

At the first arrow both feet were burned for 30 seconds in water at $100^{\circ} \mathrm{C}$. At arrow prime, the cast was removed from the left front foot and lymph flow increased immediately.

flow and the protein content was the same upon both sides. Following removal of the cast at arrow prime, the capillaries dilated promptly, the lymph flow was augmented, and the foot began to swell. The flow from the casted burned foot decreased earlier than in the non-casted burned foot. This is probably due to two facts ; first, the lymphatics in the foot without a cast, permitted to swell freely, are widely opened by the swelling and are thus harder to block by intralymphatic fibrin formation, and, second, there is much less free tissue fluid in the casted foot to enter lymphatics at any time. When coagulation of the exudate occurs, the lymphatics being of small size are more readily blocked and after clotting, there is far less free tissue fluid in the casted foot.

At varying times following burning and casting, the dye T-1824 was injected intravenously, 10 to $20 \mathrm{cc}$. of a 5 per cent solution being used. The appearance of this dye in the lymph from both feet was prompt, although it appeared in heavier concentration earlier in the burned and non-casted foot. 
From the findings upon lymph flow in the foot kept from swelling by plaster, one may conclude that the circulation has remained reasonably good and this is confirmed by the quick transfer of dye from blood to lymph.

$B$. Tissue fluid pressure. Following a burn and the resultant wide dilatation of the entire capillary bed in the burned area, the pressure in the capillaries must rise to some degree. Field, Drinker, and White (6) showed an increase in both the end lymphatic pressure and the venous pressure from the burned area immediately after burning.

End lymphatic pressures in casted and noncasted feet were measured by connecting mercury manometers to cannulas placed in lymphatics just above the burned areas. Pressures taken simultaneously were then recorded.

In the casted foot, the lymph pressure rose very rapidly and became maximum within the first 2 hours, invariably equalling systolic blood pressure. The lymph pressure fell rapidly following the attainment of the maximum pressure and was below diastolic blood pressure 2 to 7 hours after the burn. If, on the other hand, clotting of the extravascular exudate was prevented by heparinization of the animal prior to burning, the lymph pressure closely followed the systolic blood pressure over a period of 7 hours after burning, showing no tendency to fall unless there was a fall of systolic blood pressure. This finding is consistent with the fact that, coagulation being prevented by heparin, there is a continuous but small body of incompressible fluid from the blood capillaries to the cannula recording end pressure in a draining lymphatic trunk and the lymphatic channel is not even partially obstructed by clot formation.

In the non-casted burned foot, the lymph pressures rose much more slowly, corresponding to the rate of swelling of the foot. The maximum pressure was reached 3 to $4 \frac{1}{2}$ hours after burning and this never exceeded the diastolic blood pressure. Heparinization of the animal did not materially change the picture, the pressure not rising above the diastolic level. Both with and without heparinization, the tissues in the foot unsupported by a cast possess distensibility of considerable degree and failure of lymph pressure to reach systolic blood pressure simply reflects the escape of fluid into the tissue reservoir which will continue more or less open at the margins of the burn, even in the presence of the progressive coagulation which occurs when heparin is not used.

These observations are of fundamental importance since they indicate that the walls of the rigid cast literally become the walls of the blood vessels and lymphatics enclosed within. It is shown quite clearly that systolic blood pressure is exerted in the passage of fluid from the dilated capillaries into the subcutaneous tissue and lymphatic capillaries. Between the rigid wall of plaster on the outside of the burned foot and the blood within the capillaries, there is an incompressible fluid medium. Blood flows uninterruptedly through these vessels and even if their walls have been rendered extremely permeable by heat, they still operate as channels for blood flow and retain practically all the plasma, since there is no way for an abnormal volume of fluid to pass the capillary walls on account of the barrier imposed by the cast.

As shown in Figure 3, there is some increase in lymph flow from a burned, casted foot. But lacking ability to swell, which results in wide opening of lymph capillaries, and lacking either motion or massage of the part, the entrance of tissue fluid into lymphatics is slow and the amount of lymph moved is comparatively slight.

C. Arterial and venous oxygen in the blood. In any site of inflammation where the circulation is unobstructed, the increased volume and rate of blood flow will be reflected in the slight loss of oxygen which the blood incurs as it pours through the capillaries. We have made frequent examinations of the oxygen content of the arterial blood and of the venous blood collected immediately adjacent to severe burns in dogs anesthetized with nembutal. In these examinations, we have burned both fore or hind feet simultaneously and have at once encased one foot in plaster and permitted the other to swell freely without applying a dressing of any sort.

Typical results for a single animal are shown in Table I. Obviously, the oxygen in the venous blood of the burned feet, whether casted or not casted, is high and is similar in the 2 feet. This indicates unimpeded blood flow upon both sides and is evidence that the presence of a skin-tight cast does not injure the circulation. 
TABLE I

Arterial and venous oxygen in the blood of the feet of a dog burned similarly, one foot being at once encased in plaster and the companion foot untreated. Air breathed. Control blood taken from unburned hind foot

\begin{tabular}{|c|c|c|c|c|c|c|}
\hline \multicolumn{4}{|c|}{ Arterial oxygen } & \multicolumn{3}{|c|}{ Venous oxygen content } \\
\hline Time & $\begin{array}{l}\text { Con- } \\
\text { tent }\end{array}$ & Capacity & $\begin{array}{l}\text { Satur- } \\
\text { ation }\end{array}$ & $\begin{array}{c}\text { Control } \\
\text { foot }\end{array}$ & $\begin{array}{l}\text { Burned } \\
\text { casted } \\
\text { foot }\end{array}$ & $\begin{array}{l}\text { Burned } \\
\text { non-casted } \\
\text { foot }\end{array}$ \\
\hline hours & $\begin{array}{l}c c . \text { per } \\
100 \text { cc. }\end{array}$ & $\begin{array}{l}\text { cc. per } \\
100 \text { cc. }\end{array}$ & $\begin{array}{l}\text { per } \\
\text { cent }\end{array}$ & $\begin{array}{l}c c . \text { per } \\
100 \text { cc. }\end{array}$ & $\begin{array}{l}c c \text { per } \\
100 \text { cc. }\end{array}$ & $\begin{array}{l}c c . \text { per } \\
100 \text { cc. }\end{array}$ \\
\hline 0 & 15.93 & 18.15 & 87.8 & 14.71 & & \\
\hline 12 & 21.05 & 22.52 & 93.5 & 15.95 & 20.35 & 20.67 \\
\hline 24 & 19.52 & 21.51 & 90.8 & 10.67 & 17.51 & 19.22 \\
\hline
\end{tabular}

D. Skin temperatures. The skin temperature of a burned foot was measured by fixing a small thermocouple to the dorsum of a foot that had been shaved and burned immediately before. The foot was cast in dental plaster which was then dried and the temperature followed for as long as 13 days. During the period of plaster drying, which was done under a stream of air from an electric fan, the temperature of the foot dropped. This lowered temperature was held as long as the plaster remained moist and evaporation continued. It could be repeated at will by simply moistening the plaster. After the cast had dried, the temperature of the foot rose rapidly to, and often slightly above, that of the unburned feet and remained so throughout the course of the experiment. A failure of the capillary circulation would occasion an immediate fall of the temperature in the part affected.

\section{Experiments in Group 2}

The efficacy of the closed-plaster method in the treatment of burns.

Dogs chosen for these experiments were in good health and, as a rule, of quiet disposition. Nembutal anesthesia was invariably used, and during the first few experiments the animals were kept anesthetized for 24 to 36 hours to avoid the possibility of pain following their return to consciousness. This was soon found to be unnecessary, for as soon as the dogs awoke they walked about on the cast and gave no evidence of discomfort. They were then confined to cages sufficiently large to move about in but not so large as to encourage walking on the casted foot.

In the first animals, dental plaster, re-enforced by a metal shell, was used, just as was the case in the experiments designed to test the patency of the circulation. Burns were produced, ranging from 15 seconds' immersion in water at $80^{\circ} \mathrm{C}$. to 45 seconds at $100^{\circ} \mathrm{C}$. A burn of 15 seconds at $100^{\circ} \mathrm{C}$. is sufficient to produce sloughing of the skin. The skin-tight plaster was removed at varying intervals from 24 hours to 17 days after the initial application. Rectal temperatures were taken daily. In some cases, white cell counts, plasma protein concentrations, and hematocrit determinations were made daily, and in all instances, at least once a week. Occasionally, after the first 24 hours, the white blood count and temperature would show a sharp rise and then return to normal. In the event of infection later in healing, there was another rise in white blood count and temperature.

An interesting observation was the prompt swelling of the burned foot upon removal of the plaster cast. As a rule, this did not occur after the 6th day, even with a severe burn (30 seconds' immersion in water at $100^{\circ} \mathrm{C}$.), unless infection was present. Interest in the persistence of the vaso-dilator reaction, so fundamental in inflammation, is long standing. The phenomenon is of vital importance in the closed-plaster treatment of burns, since, if a burned part is encased in plaster in order to prevent swelling and capillary leakage, one needs to know how soon the dressing may be removed without the probability of permitting the very contingency the plaster cast is designed to prevent. One cannot make a definite prediction upon the matter, but it has been our practice, if casts were removed within a week after burning, to be prepared for prompt reapplication of plaster and even to use a tourniquet to control blood flow if the cast was opened near enough to the time of burning to be sure that swelling would immediately begin if opportunity was permitted.

The use of the skin-tight plaster cast resulted in complete control of swelling without endangering the circulation in the part during a continuous application of 9 to 14 days. At the end of this time, in burns over 15 seconds in water at $80^{\circ} \mathrm{C}$., there was some sloughing of tissue, a varying amount of infection, and swelling, following the removal of the cast. None of the animals was allowed to survive over 24 hours after the removal 
of the cast, except those burned for 15 seconds at $80^{\circ} \mathrm{C}$. In the latter case, upon removal of the plaster at 10 to 12 days, no sloughing of skin, no infection, and no swelling after removal was noted. Sections of skin, subcutaneous tissue, and muscle from the burned feet could not be distinguished from similar tissues from a normal foot of the same animal. Such a burn, 15 seconds' immersion at $80^{\circ} \mathrm{C}$., is severe enough, however, if left uncasted, to produce marked swelling, blisters, and weeping within a few hours.

Convinced that the principle of the closed-plaster treatment was sound, we sought to revise it in order to make the method more practical. The feet were burned in the same manner as previously described. Following this, 3 to 4 layers of thin, sterile, dry gauze were wrapped gently around the foot and held in place with one layer of gauze bandage. A roller-plaster bandage was then applied over this primary gauze covering but with absolutely no pressure. As seen in Figure 4 (B and $\mathrm{C}$ ), the gauze dressing and plaster extended well above the upper margin of the burn. The plaster was then dried thoroughly and the animal allowed to walk about as soon as he recovered from the anesthesia. In these animals, the burning period ranged from 15 seconds' immersion in water at $80^{\circ} \mathrm{C}$. to 45 seconds at $100^{\circ} \mathrm{C}$. As in the previous group, white blood cell counts, plasma protein, and hematocrit determinations were made at intervals.

In the more severely burned feet, the destroyed epithelium began to slough off at about the 14th to 16 th day, leaving under it a thin new layer of epithelium that had regenerated from the deep hair follicles and sebaceous glands not destroyed by even a very severe burn (45 seconds' immersion in water at $100^{\circ} \mathrm{C}$.).

The regenerated epithelium was thin and delicate and the reapplication of plaster to this, for a week or two, by the dry gauze, roller-bandage method prevented the subepithelial petechial hemorrhages and the cracking of the thin epithelium characteristically seen when the part was left unsupported. A most striking feature about the foot healed as a result of the plaster treatment was the lack of scar tissue in the subepithelial area. The epithelium was soft and pliable and the structures beneath it were clearly defined.
There was no evidence of contracture and the toes remained freely moveable.

In Figure 5, the right hind foot of an anesthetized $\operatorname{dog}$ was burned for 30 seconds in water at $100^{\circ} \mathrm{C}$. A roller-plaster clressing was immediately applied as in Figure 4 . Six days later the dressing was removed and the foot inspected (Figure 5, B). A tourniquet had been applied above the ankle to prevent swelling of the foot during the removal. This was subsequently found unnecessary when infection was absent, the capillaries, at least to a large degree, having recovered their normal tone or having become thrombosed. Twelve days after the burn (Figure 5, C), the dressing was again removed. Figure 5 (D) shows the same foot, 7 hours later, with no evidence of infection or swelling. The roller-plaster bandage was not reapplied and the dog was allowed to rum about freely. Subsequent photographs (Right foot, Figure 6, E, F, G, and H) show the process of recovery. Re-epithelialization was complete at 28 days (Figure $6, \mathrm{E}$ ).

In $\mathrm{F}$ (Figure 6), the left foot, heretofore normal, was burned 3 hours previously, by immersion in water at $100^{\circ} \mathrm{C}$. for 30 seconds. The degree and extent of the burn were exactly those used for the right foot but after the burn no plaster dressing was used. Photograph $G$ shows the tremendous swelling of this foot with beginning regeneration of epithelium which was present 21 days after the burning and $H$, the condition at 52 days.

The epithelium regenerated from between the toes and from above the margin of the burn. In contrast to the roller-plaster-treated foot, the epithelium was thicker and the subcutaneous tissue dense and fibrous. The important lesson from this experiment is the making of a 3rd-degree burn out of an obviously deep 2nd-degree lesion by destruction of the hair follicles and sebaceous glands either through the accumulation of a large amount of clotted, subcutaneous exudate under pressure, local infection, or both factors working together.

\section{DISCUSSION}

Heat may produce only physiological dilatation and permeability of the capillaries through axone reflexes. This results from a painful stimulus to 


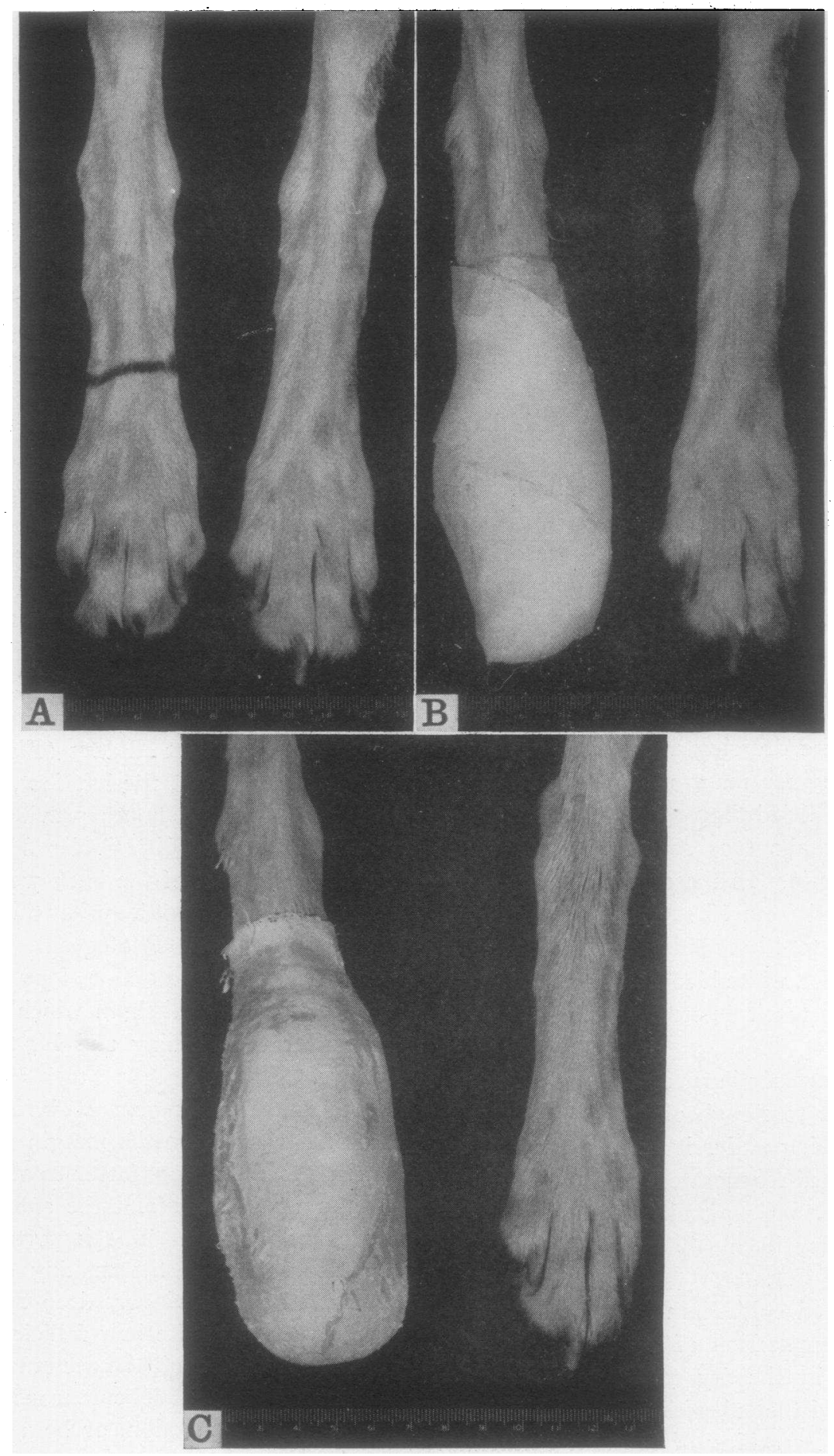

Fig. 4. The Use of a Roller-Plaster Bandage in the Treatment of a Burn

A. Hind feet of anesthetized dog prior to burning. The transverse line above the right foot indicates the upper limit immersion in water. B. The burned foot wrapped in gauze covered by one layer of bandage. C. The roller-plaster bandage in place. Note the distance above the upper margin of the burn to which the dressing extends. 


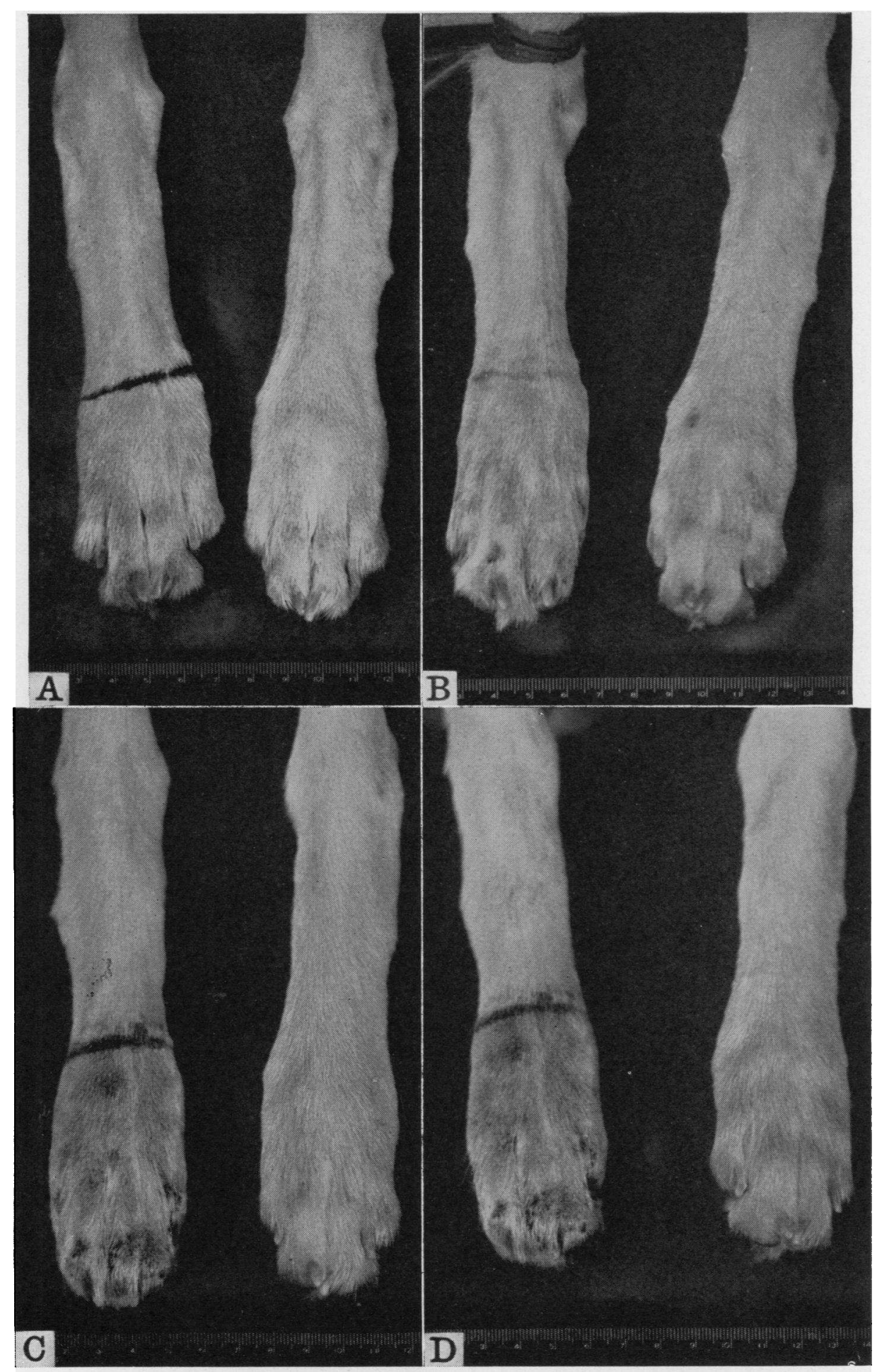

Fig. 5. The Efficiency of the Roller-Plaster Bandage Treatment

A. The feet prior to burning. Right foot marked for upper level of immersion in water at $100^{\circ}$ C. for 30 seconds. B. Six days after the burn. Note the tourniquet upon the burned leg, which was put in place before removing the roller-plaster dressing. C. Twelve days after the burn. D. Seven hours after C. Note that there has been no swelling of the burned right foot. 


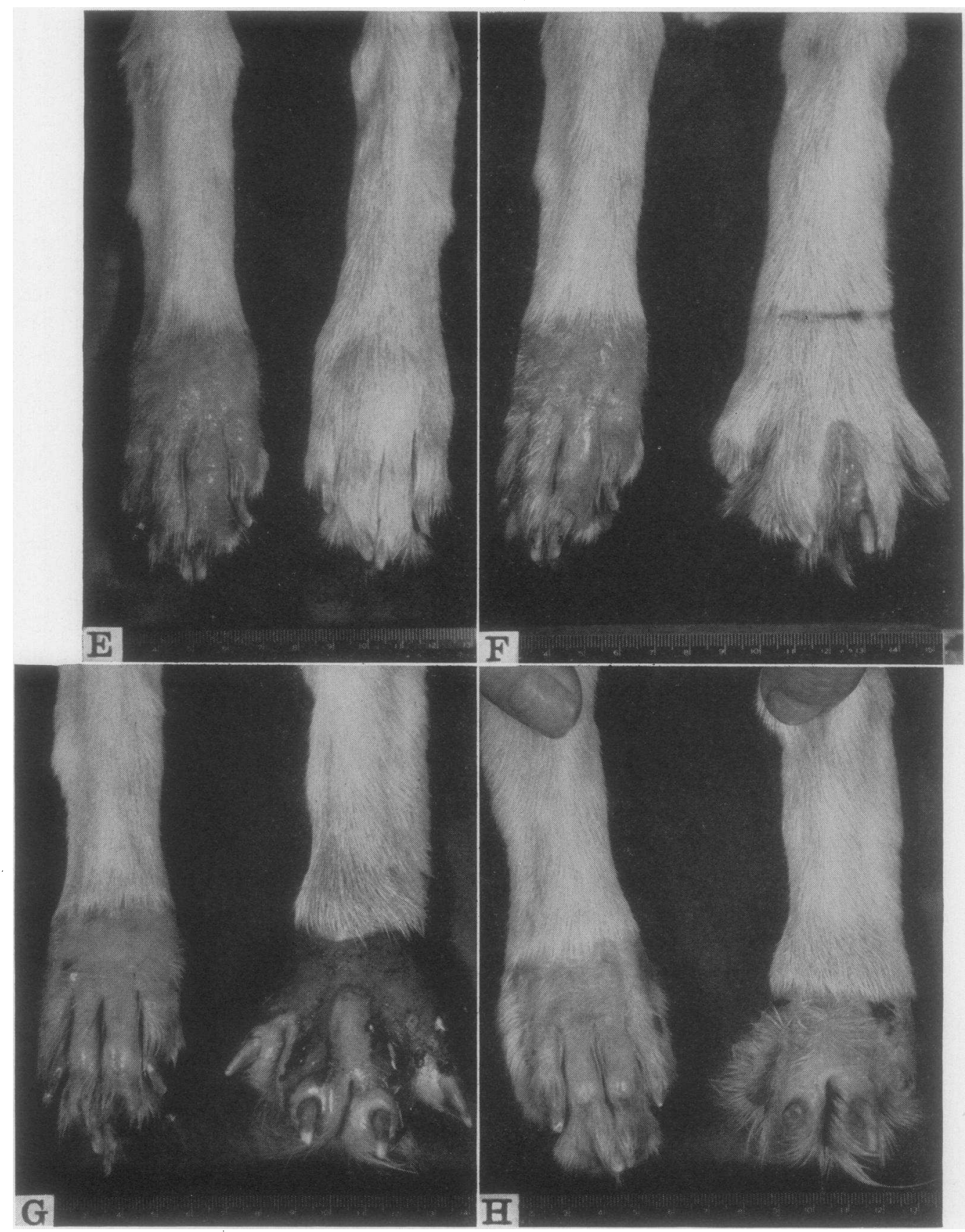

Fig. 6. Further Stages in Burn Healing Following Use of a Roller-Plaster Bandage, Compared with a Similar Burn Where Swelling Has Been Unrestrained

E. Right foot, 28 days after burning, completely re-epithelialized. F. Right foot, 34 days after burning. Left foot, immersed 30 seconds in water at $100^{\circ} \mathrm{C}, 3$ hours before taking the photograph. G. Right foot, 55 days after burning. Left foot, 21 days after burning. H. Right foot, 86 days after burning. Left foot, 52 days after burning; re-epithelialization is still incomplete, contractures have formed, and the subcutaneous tissue is dense and fibrous. 
the skin, causing local dilatation of the capillaries, arterioles, and venules. Krogh (7) demonstrated a reversible dilatation of capillaries to small increases in temperature. McCarrell (8) showed this more clearly in the nasopharynx of a dog. She found a critical temperature which limited completely reversible nervous dilatation of capillaries. If the temperature was raised above this point, the recovery of the capillaries was progressively delayed, due to damage of the capillary wall. Heat effects which produce no tissue destruction, but simple capillary dilatation and leakage without actual pathologic changes in the capillary wall, do not constitute a burn. That local dilator reflexes may cause the major part of the swelling in a minor burn is, however, clearly demonstrated by the rapid subsidence of swelling beginning within 4 hours, often seen in a foot burned for 15 seconds in water at $80^{\circ} \mathrm{C}$.

When heat, in itself, causes actual capillary trauma, capillary leakage in a burned area will continue for a long time, but the capillaries in a burned part are not all injured to the same degree. It is, therefore, impossible to predict exactly how long excessive capillary permeability will continue. A very severe burn, 2 minutes in boiling water, is sufficient to coagulate completely the whole thickness of the skin, so that a great many capillaries are immediately thrombosed. Others may be injured to the point of passive conductivity, with walls freely permeable and with thrombosis occurring later in a large number. Microscopic sections of the dog's skin after a severe burn, one that will end in a complete slough, show dilated capillaries packed with red cells just beneath the epithelium without evidence of thrombosis, indicating the presence of capillaries conducting blood through tissue which is not certainly viable.

The formation of blisters in dogs subjected to this type of experimental burn is a regular occurrence. These form in the delicate tissue between the toes and foot pads, following even a minor burn. The mechanism of formation of these blisters, and, indeed, the weeping from the area following the removal of the dead epithelial covering, depends, we believe, on seepage from tissue spaces, and possibly from small lymphatics in the subepithelial layer of the skin. Early, this seepage is small, but after exudate begins to accumulate in the subcutaneous tissue and the tissue pressure increases, the under surface of the skin is stretched and fluid is pushed upward through the separated fibers of the corium and finds its way to the surface. We have been able to demonstrate, particularly in calves, that the coagulation time of the lymph coming from a burned area is greatly prolonged after a burn. There is simultaneous prolongation of the coagulation time of lymph obtained from an unburned area. We believe that continued weeping into the subcutaneous tissue and from the surface depends, at least in part, on this fact.

The accumulation of subcutaneous edema and blister formation can be, to a large degree, prevented by heparinization of the animal prior to burning (Glenn, Peterson, and Drinker (1)). The lymph flow, instead of tapering off after 2 to 6 hours due to progressive coagulation, is maintained at a high level over many hours. By contrast, the injection of a small amount of tissue extract (thromboplastic material) into the burned foot of a non-heparinized dog results in prompt blockage of the extracellular tissue spaces and lymphatics by the immediate clotting of the extravascular exudate. Following this, the swelling of the part becomes very great and blister formation rapid, since fluid will continue to leak from injured capillaries until the part is distended as far as the capillary pressure can drive fluid into the tissues.

The idea that the best dressing for a burn should prevent capillary leakage and that this be attained by the use of a "pressure dressing" is an old one which reappears in recent literature. Mason (9), in writing on the local treatment of burns, stated: "As for the coverings for the wound, there are only two prerequisites; first, that they be non-irritant and, second, that they adequately protect the surface and prevent fluid loss from it." Since that time, large pressure dressings have been used extensively with excellent results (Siler and Reid (10)).

To Orr (11) belongs credit for the institution of certain physiological principles in the treatment of infected wounds. For more than 20 years, he campaigned for the recognition of the patient's power to recover when therapy is carried out with simple physiologic principles in mind, and the 
closed-plaster treatment is a physiological measure in many ways, some of which extend beyond the matters we have discussed. For example, the control of absorption of toxic products, either bacterial or metabolic, depends to a large degree upon the decreased lymphatic circulation brought about by immobilization of the part in plaster. Barnes and Trueta (12) have performed a number of interesting experiments on the effect of immobilization in plaster on absorption of bacterial toxins, snake venom, strychnine, and tetanus toxin, injected subcutaneously. Their studies show beyond doubt that provided the part is immobilized, the absorption of lethal doses of these poisons is so slowed as to prevent death from occurring. They likewise applied plaster casts to the feet of burned animals and pointed out that the subcutaneous edema could be checked by this method. With regard to burns, it should be borne in mind that the toxic products may be of such small molecular size as to be absorbed directly into the blood and while immobilization in plaster may check this in some degree, it is essentially a means of controlling lymphatic absorption.

The clinical use of the plaster dressing has been reported by Löhr (13) who employed this dressing extensively. He stressed the primary application of cod liver oil ointment, and he also recognized the immobilizing effect of the closed-plaster dressing. He believed that keloid formation was to a large extent prevented by this method. More recently, Roulston (14) treated 16 cases, a total of 26 limbs, consisting of 11 hands and wrists, 3 wrists, 8 feet and ankles, and 4 complete upper extremities, by the closed-plaster method. The earliest case treated by him was 17 hours after the burn. The rest of the cases were 4 days or more after the initial injury. All were infected at the time of application. $\mathrm{He}$ concluded that the results indicated a more rapid epithelialization and return of function than in cases treated by other methods. Stonham (15) in India, treating old infected burns by closedplaster, found more maceration of the tissue and persistent infection than when the wounds were left open.

These different results are related to our examination of the closed-plaster treatment of burns, though often indirectly. Their importance consists mainly in their disclosure of the fact that men actively engaged in surgical practice have found the method "works," though the reasons given to explain success are not infrequently unsound, missing the physiological perfection with which closed-plaster dressings meet the needs of the situations encountered.

So far as our experimental observations are concerned, there are certain points which should instill caution in the minds of clinicians. The findings we have reported have been repeated many times. They are entirely true for the controlled experimental conditions we have been able to impose in the laboratory and they are ready to be carried into the clinic if seasoned with the judgment that transition always requires.

Viewed critically we suggest that the following facts be remembered :

1. We have reported on the use of closedplaster applied immediately after burning with a severity we have learned to evaluate fairly well. The treatment anticipated swelling and prevented it. While, even in civil life, more human burns are available for treatment soon after the injury than we had supposed would be possible, they are never so free of capillary leakage and swelling as in the case of our dogs. Experience does not permit us to do more than suggest that, even if there has been delay in application, closed-plaster will do no harm and may help to check surface oozing, enhanced by plasma administration or by secondary inflammation due to infection. Obviously, the arms and legs are the ideal parts for this treatment and since closed-plaster may do much to reduce eventual deformities, burns of the extremities should receive the promptest possible attention.

2. Dog skin subjected to a burn, due to the insulating effect of the hair and to greater natural density, does not crack and peel as easily as that of man. Nor is the dog anywhere near so susceptible to bacterial infection, particularly streptococcic infection. Consequently, our experiments have dealt with animals where surface weeping of fluid and infection have been far less troublesome than in human burns. There is no reason why measures for the control of infection, such as local application of the sulfonamides, in concentrations high enough for local bacteriostatic control but sufficiently low to prevent the absorption 
of toxic amounts of the drug, should not accompany the use of plaster. Finally, the dressing is easy to remove and there is never any contraindication to its removal except the possible disadvantage to the patient.

3. Some oozing from burned surfaces will always be encountered. This is absorbed to a small degree by the thin layer of gauze under the plaster and even more by the plaster itself. The dressing has the two-fold virtue of keeping the part at the volume existing when it was applied and, at the same time, reducing tissue maceration by absorption of some of the exudate. It is well to keep in mind that evaporation from the plaster may be increased by a stream of air played over it and this has the advantage of cooling the burned area under the plaster. We have not tried other materials than plaster for forming the dressing. It may be that another substance can be found which is as easy to apply as the plaster bandage, does not shrink during drying, is lighter, tougher, and unaffected by water. Whether or not absorption of fluid by plaster, after setting, is certainly advantageous can only be learned by trial in the laboratory and then in the clinic.

4. The most serious danger from the use of the closed-plaster dressing is interference with the circulation. It is particularly vital this should not occur in a lesion which contains tissue already damaged but not killed and presumably overdependent on oxygen supply for recovery. When the circulation to a burned extremity is blocked by a rigid dressing, we believe the accident will generally be due to failure in realizing the principles which must be observed in the use of rigid encasement of parts swelling when the dressing is applied and certain to attempt to swell for a week or more after the dressing is in place.

It is noticeable that we have avoided the use of the term "pressure dressing" and have emphasized that in the use of roller-plaster bandages, the dressing of itself should not add an increment of pressure. In a burn treated some hours after the injury and already at the limit of swelling, this avoidance of external pressure may not be so vital, but even then it should be borne in mind that clotted exudate will not be eliminated to any appreciable degree by pressure from the dressing and the danger of impairing the capillary circulation by pressure should not be forgotten. The closed-plaster technique is used to prevent swelling and capillary leakage, not to reduce swelling. It is applied sufficiently beyond the margins of the burn to include all capillaries which the injury may have caused to leak abnormally, or in which leakage may be induced. Within the boundaries of the dressing are all the capillary venules and the veins which drain the part. The dilatation of arterioles, arterio-venous anastomoses, and capillaries, which occurs promptly following a burn, means that all pathways for the movement of blood from arteries to veins, and still capable of reacting, are open and conducting blood. This maximum physiological blood supply increases the pressure in capillary venules and veins and, with the attachment of their walls to other tissues in the neighborhood by strands of fibrous tissue, causes the channels for blood flowing out of the burned region to stay open. This has been shown by the fact that the saturation of venous blood from burned extremities, enclosed or not enclosed in plaster, is comparable to that of the arterial blood. There is an increase in tissue pressure all through the part involved by the burn, but this is distributed uniformly through the incompressible watery medium held within the plaster. No danger of circulatory occlusion will occur if this fact is kept in mind, but if it is not, and closedplaster is used unintelligently, serious trouble may readily develop.

Figure 7 illustrates a possibility which may readily arise. In this case, a 2 -inch circular burn of 30 seconds' duration from water at $100^{\circ} \mathrm{C}$. has been made halfway up the foreleg and a skintight cast applied at once, as shown in the photograph. The foot was uninjured and the local circulation was not directly involved in the burn higher in the leg, but venous obstruction with the gradual development of edema occurred in the foot due, initially, to the fact that the blood from the foot, in order to move on, had to enter veins where the pressure was abnormally high. Stasis followed throughout the vessels below the cast, which grew worse as edema and anoxia progressed. Such a result would not have occurred had the cast extended down and surrounded the foot. In that case, no swelling with increased stasis and interference with oxygen supply could take place and healing would have progressed without complications. It is absolutely necessary 


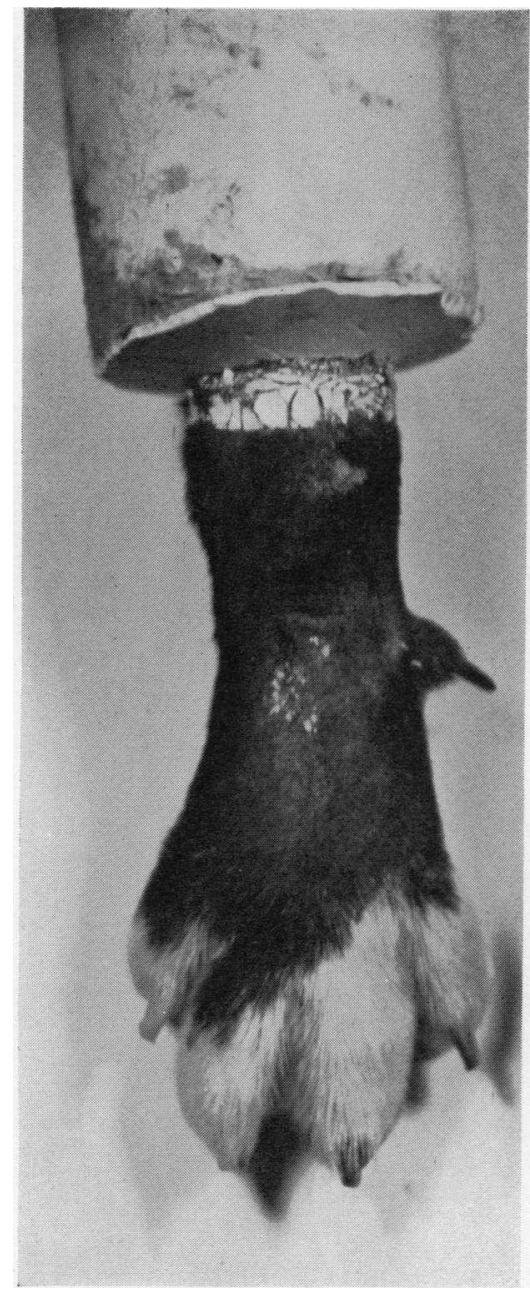

Fig. 7. Illustrating Error in Closed Plaster Treatment of Circular Burn of Leg; the Foot is Badly Swollen

This can be prevented by extending the plaster dressing to enclose the foot as well as the burned area above it.

to pay the strictest attention to the part distal to a burn on an extremity. It must, like the burned area, be completely enclosed in the plaster dressing.

5. One of the features of the closed-plaster treatment of burns in dogs which surprised us, though we knew it as a possibility, was the freedom from pain which resulted. In our opinion, this is due not simply to the exclusion of air from the burned surface, but to the prevention of rapid swelling which causes stretching and tearing of fine nerve fibers. Whatever the physiological reason for the relief given through immobilization of a burned part in a plaster dressing, the result, indicated experimentally, is not the least important of the benefits conferred by the method. Whether this feature of the treatment, so evident in dogs, will be equally noteworthy in man must await clinical observation.

6. One of the lessons we have learned from these experiments is the impossibility of predicting, until some time after burning, just how much damage the burn itself has caused. As a rule, more cells survive the immediate effects of the heat than we have believed could possibly be the case. It is startling to find that the immersion of the foot of a dog in water at $100^{\circ} \mathrm{C}$. for 45 seconds does not destroy the entire epithelium. If the part is enclosed promptly in plaster so that capillary leakage is restrained, and if severe infection does not occur, the burned part may heal in 3 to 4 weeks, the epithelium being regenerated from deeply-lying parts of the hair follicles and sebaceous glands. We believe that the closedplaster technique does its greatest service by holding the environment of the cells in burned tissue as nearly normal in volume and composition as can at present be attained. This accomplishment is expressed in the preservation of injured cells which will die if given the additional insult of an abnormal environment. When a burned extremity is to be encased in plaster, the surgeon does not face the conventional task of putting on a dressing for temporary coverage of such tissue as may survive; he will apply treatment which must operate as an active agent in saving viable cells. The application of the dressing requires all the care and sagacity which are used in a surgical operation and can be no matter for routine measures by subordinates.

7. In dogs, the hairiness and density of the skin prevents most of the early separation of the epithelial layer which, though dead, acts as a protective coating to the underlying living cells. It separates in due course of time with no damage to the tissue beneath, and while in place prevents bacterial invasion and even places some check upon the oozing of fluid. Debridement of this epithelial covering has never proved wise in dogs, and even though human skin may not be so durable following a bad burn, it is probable that premature debridement will prove harmful. 


\section{SUMMARY}

The authors take the position that the fundamental condition to be met in treating burns is increased capillary permeability. This results in reduction of blood volume which is met by intravenous injections of blood plasma. The local consequences of abnormal capillary leakage have not been properly appreciated and, therefore, have not been combated by physiological methods of treatment. These consequences are:

1. Loss of plasma into the tissues surrounding the burn.

\section{Swelling.}

3. Coagulation of the plasma in and about the burned area, resulting in a mass of abnormal material which must be removed during healing and which provides culture medium for bacterial growth and induces extensive deformity through excessive formation of fibrous tissue.

The enclosure of the burned part in a plaster of Paris dressing prevents abnormal leakage from the injured capillaries, since the rigid plaster case by virtue of the incompressible watery medium between it and the blood vessels becomes, in fact, the capillary wall and thus meets the necessities of the situation physiologically.

Experiments are reported in which the feet of clogs were burned for varying periods by immersion in hot water. They were then enclosed in plaster; first, by casting the burned foot which was held deep in liquid plaster until setting occurred, and second, by the use of roller-plaster bandages.

It is shown that the blood flow through the burned feet treated by these methods remained excellent and that healing occurred more rapidly and with less deformity than could be obtained if capillary leakage was unrestrained, as is the case in the absence of a rigid enclosure of the part.

The physiological reasons for this success are analyzed and, at the conclusion, the reservations are listed which should be in the minds of clinicians when the closed-plaster technique is used in treating human burns.

Inclusion of this paper in this number of the Journal of Clinical Investigation was made possible by the Josiah Macy, Jr. Foundation, at the request of the editors, in order to provide prompt publication of results of investigation particularly relevant to military medicine.

\section{BIBLIOGRAPHY}

1. Glenn, W. W. L., Peterson, D. K., and Drinker, C. K., The flow of lymph from burned tissue, with particular reference to the effects of fibrin formation upon lymph drainage and composition. Surgery, 1942, 12, 685.

2. Underhill, F. P., Fisk, M. E., and Kapsinow, R., Studies on the mechanism of water exchange in the animal organism: III. The extent of edema fluid formation induced by a superficial burn. Am. J. Physiol., 1930, 95, 325.

3. Harkins, H. N., Experimental burns: I. The rate of fluid shift and its relation to the onset of shock in severe burns. Arch. Surg., 1935, 31, 71.

4. Blalock, A., Wilson, H., Weinstein, B. M., and Beard, J. W., Loss of protein from the blood stream. Effects of the injection of solution of pituitary and of epinephrine. Arch. Surg., 1933, 26, 330.

5. Menkin, V., Studies on inflammation. IX. A factor in the mechanism of invasiveness by pyogenic bacteria. J. Exper. Med., 1933, 57, 977.

6. Field, M. E., Drinker, C. K., and White, J. C., Lymph pressures in sterile inflammation. J. Exper. Med., 1932, 56, 363.

7. Krogh, A., The Anatomy and Physiology of Capillaries. Yale University Press, New Haven, 1929, page 336.

8. McCarrell, J. D., The effect of warm and of cold nasopharyngeal irrigation on cervical lymph flow. Am. J. Physiol., 1940, 128, 349.

9. Mason, M. L., Local treatment of the burned area. Surg., Gynec. and Obst., 1941, 72, 250.

10. Siler, V. E., and Reid, M. R., Clinical and experimental studies with the Koch method of treatment of heat burns. Ann. Surg., 1942, 115, 1106.

11. Orr, H. W., The physiologic factors involved in protecting the patient against infection in the healing of fractures in compound wounds. Tr. and Stud., Coll. Physicians, Philadelphia, 1943, 10, 187.

12. Barnes, J. M., and Trueta, J., Absorption of bacteria, toxins and snake venoms from the tissues. Importance of the lymphatic circulation. Lancet, 1941, $1,623$.

13. Löhr, W., Die Behandlung von frischen und älteren Hand- und Fussverletzungen mit Substanzverlusten mit dem Lebertran-Gipsverband. Chirurg., 1934, 6, 5.

Löhr, W., Die Behandlung grosser, flächenhafter Verbrennungen 1., 2. und 3. Grades mit Lebertran. Chirurg., 1934, 6, 263.

Löhr, W., Über die Lebertransalbenbehandlung (mit und ohne Gipsverband) bei frischen Verletzungen, Verbrennungen und phlegmonösen Entzündungen. Zentralbl. f. Chir., 1934, 61, 1686.

14. Roulston, T. J., Closed-plaster treatment of burns of the extremities. Brit. M. J., 1941, 2, 611.

15. Stonham, F., Closed plaster for burns of limbs. Brit. M. J., 1942, 1, 737. 